\title{
Misuse of Competencies in Pharmacy Curriculum: The Spain Case Study
}

\author{
Ines Nunes-da-Cunha1, Fernando Fernandez-Llimos ${ }^{2, *}$ \\ ${ }^{1}$ Doctoral Programme in Pharmacy, Social Pharmacy, Faculty of Pharmacy, University of Granada, Campus de Cartuja, Calle del Prof. \\ Clavera, Granada, SPAIN. \\ ${ }^{2}$ Department of Social Pharmacy, Faculty of Pharmacy, University of Lisboa, Avda. Prof. Gama Pinto, Lisbon, PORTUGAL.
}

\begin{abstract}
Background: Pharmacy curriculum must prepare students with the necessary competencies to respond to society health-related needs. Aim: This study aims to analyze the allocation of competencies that pharmacists should acquire during their education in the courses constituting pharmacy curricula in Spain. Materials and Methods: All mandatory undergraduate pharmacy courses from all Spanish universities were analyzed in accordance with the official Spanish competency framework. Information about courses and competencies assigned was extracted from the syllabi available at the websites of all colleges of pharmacy existing in Spain (2016/2017). Elective courses, courses that correspond to the internship period or final dissertation activities and courses that did not present an online syllabus were excluded from the analysis. The allocation of the 15 general competencies and 67 specific competencies defined in the official Spanish competency framework was investigated. Results: The 22 Spanish universities offering pharmacy degrees teach 1261 courses, of which 942 are mandatory courses. Syllabi of 881 courses were available on the internet and were analyzed. A total of 560 could be objectively associated with the following areas of knowledge: $23.8 \%$ with chemistry, $6.8 \%$ with physics and mathematics, $16.4 \%$ with biology, $13.0 \%$ with pharmaceutical technology, $29.6 \%$ with medicine and pharmacology, $10.2 \%$ with legislation and social pharmacy and $0.2 \%$ with internships (not taught during the internship period). Competency allocation patterns are very different across universities. Conclusion: The results show that Spanish colleges of pharmacy do not appropriately use the official Spanish competency framework. Competencies and courses are mismatched in pairing basic sciences to practice competencies.
\end{abstract}

Key words: Competencies, Competency framework, Curriculum, Pharmacy colleges, Spain.

\section{INTRODUCTION}

Competency-based education is increasingly common among health professionals. ${ }^{1,2}$ The development of competency frameworks for pharmacy education and practice has emerged worldwide and they have been used especially in the design, development and revision of pharmacy curricula. ${ }^{3}$ During their education and training, pharmacy students must achieve the necessary competencies that allow them to respond to patient and population health-related needs. ${ }^{4}$ A competency-oriented curriculum must align the competencies to be achieved with teaching-learning methodologies, educational practices, different contexts and learning settings, assessment methods and research activities. ${ }^{1}$ Pharmacy curricula should also follow this principle. ${ }^{5}$

The International Pharmaceutical Federation (FIP), in partnership with the World Health Organization (WHO) and UNESCO, created the global competency framework with the aim of supporting the educational development of pharmacy practitioners. ${ }^{6}$ In Europe, a competency framework for
Submission Date: 03-02-2019; Revision Date: 29-03-2019; Accepted Date: 04-09-2019

DOI: 10.5530/ijper.53.4.123 Correspondence:

Prof. Fernando FernandezLlimos,

Department of Social Pharmacy, Faculty of Pharmacy, University of Lisboa, Avda. Prof. Gama Pinto, Lisbon, PORTUGAL.

Phone: +351-217946 400 E-mail: f-llimos@ff.ul.pt

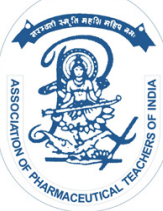

www.ijper.org 
pharmacy education and training has also been developed to be used as a quality assurance system. ${ }^{7,8}$ Australia, ${ }^{9}$ Brazil, ${ }^{10,11}$ Canada, ${ }^{12}$ Ireland, ${ }^{13}$ New Zealand,,${ }^{14}$ Portugal, ${ }^{15}$ Singapore, ${ }^{16}$ Spain,${ }^{17}$ Thailand, the United Kingdom ${ }^{18}$ and the United States ${ }^{19}$ are examples of countries that have also established their own competency frameworks. After signing the Bologna Declaration in 1999, which established the harmonized European Higher Education Area (EHEA), Spain created its own pharmacy education competency framework. The CIN/2137/2008 Ministerial order established the duration of the pharmacy degree, the requirements of the curriculum and the competencies that qualify pharmacists for the practice of the profession. ${ }^{17}$ The curriculum has a duration of 300 European Credits (ECTS: European Credit Transfer and Accumulation System) and the students must acquire at least 15 general competencies (Table 1) and 67 specific competencies divided by 7 areas of knowledge. A total of 210 ECTS are assigned to the first six areas of knowledge, which correspond to the following: chemistry (54 ECTS); physics and mathematics
(12 ECTS); Biology (42 ECTS); pharmaceutical technology (24 ECTS); medicine and pharmacology (66 ECTS); and legislation and social pharmacy (12 ECTS). The seventh area of knowledge corresponds to the supervised internship and to the final dissertation, with 30 ECTS assigned. ${ }^{17}$ The inclusion of elective courses in the curriculum is also possible, which can provide students with other competencies beyond those mentioned in the CIN/2137/2008 Ministerial order.

Using Spain and the CIN/2137/2008 Ministerial order as a case study, this study aims to analyze the quality of the competency allocation (i.e., general and specific) to courses in the pharmacy curricula of Spanish universities.

\section{MATERIALS AND METHODS}

A list of colleges of pharmacy in Spain was extracted from the website of the Spanish Pharmacist Association [Consejo General de Colegios Oficiales de Farmacéuticos]. ${ }^{20}$ Each Spanish college of pharmacy website was analyzed to obtain the following information: location; public or

\begin{tabular}{|c|c|}
\hline $\begin{array}{l}\text { Competency } \\
\text { Number }\end{array}$ & Description \\
\hline GC 1 & $\begin{array}{l}\text { Identify, design, collect, analyze, control and produce drugs and medicines and other products and raw materials of } \\
\text { medical interest for human or veterinary use. }\end{array}$ \\
\hline GC 2 & Evaluate therapeutic and toxic effects of pharmacologically active substances. \\
\hline GC 3 & $\begin{array}{l}\text { Learn to apply the scientific method and acquire skills in handling legislation, sources of information, literature, } \\
\text { protocol development and other aspects that are necessary considered for the design and critical assessment of } \\
\text { preclinical and clinical trials. }\end{array}$ \\
\hline GC 4 & Design, prepare, deliver and dispense medicines and other health products of interest. \\
\hline GC 5 & $\begin{array}{l}\text { Provide therapeutic counseling in pharmacotherapy and diet therapy, as well as in the nutritional and food field in the } \\
\text { establishments in which they provide services. }\end{array}$ \\
\hline GC 6 & $\begin{array}{c}\text { Promote the rational use of medicines and health products, as well as acquire basic knowledge in clinical } \\
\text { management, health economics and efficient use of health resources. }\end{array}$ \\
\hline GC 7 & Identify, evaluate and assess drug-related problems, as well as participate in pharmacovigilance activities. \\
\hline GC 8 & Carry out clinical and social pharmacy activities, following the pharmaceutical care cycle. \\
\hline GC 9 & $\begin{array}{l}\text { Engage in health promotion activities, disease prevention, at the individual, family and community level; with an } \\
\text { integral and multi-professional vision of the health-disease process. }\end{array}$ \\
\hline GC 10 & $\begin{array}{l}\text { Design, apply and evaluate reagents, methods and clinical analytical techniques, knowing the basics fundamentals } \\
\text { of clinical analysis and the characteristics and contents of laboratory diagnosis reports. }\end{array}$ \\
\hline GC 11 & Evaluate the toxicological effects of substances and design and apply the corresponding tests and analyzes. \\
\hline GC 12 & Develop hygienic-sanitary analysis, especially those related to food and the environment. \\
\hline GC 13 & $\begin{array}{l}\text { Develop communication and information skills, both oral and written, to deal with patients and users of the center } \\
\text { where they perform their professional activity. Promote the capacities of work and collaboration in multidisciplinary } \\
\text { teams and those related to other health professionals. }\end{array}$ \\
\hline GC 14 & $\begin{array}{l}\text { Know the ethical and deontological principles according to the legislative, regulatory and administrative dispositions } \\
\text { that govern the professional practice, understanding the ethical implications of the health in a social context in } \\
\text { transformation. }\end{array}$ \\
\hline GC 15 & $\begin{array}{l}\text { Recognize one's own limitations and the need to maintain and update professional competence, paying special } \\
\text { attention to the self-learning of new knowledge based on the available scientific evidence. }\end{array}$ \\
\hline
\end{tabular}

Abbreviation: GC = General Competencies. 
private condition; the curricular plan for the academic year 2016/2017 (i.e., the list of all courses, distribution by degree year and ECTS attributed); and a list of the CIN/2137/2008 competencies assigned to each course of the curriculum. Additionally, for each college of pharmacy, the following information about all curriculum courses was collected: the course name; its distribution throughout the degree; whether the course is mandatory or elective; whether the course is related to the internship and final dissertation; the number of ECTS attributed; and the presence of the course syllabus on the college website. Courses without online syllabi, elective courses and courses that specifically focus on the internship period or final dissertation were excluded. As in a previous worldwide analysis, a syllabus was considered sufficient for the analysis if contained a description of the objectives of the course (including skills, knowledge and attitudes to provide), course learning outcomes (including the competencies assigned), complete educational contents to teach in the course and students' assessment methods. ${ }^{21}$

All courses were analyzed in accordance with the competencies defined by the CIN/2137/2008 Ministerial order: 15 general competencies and 67 specific competencies grouped into the 7 areas of knowledge (chemistry; physics and mathematics; biology; pharmaceutical technology; medicine and pharmacology; legislation and social pharmacy; internship and final dissertation). A database was created, including each course and the general and specific competencies that were paired in the course syllabus. To objectively identify into which of the 7 areas each course should be classified, the proportion of the specific competencies of each the seven areas assigned to that course was calculated. The course was allocated to the area with the highest percentage of specific competencies assigned. In the case of a tie between two areas, the main area of knowledge was classified as non-specific.

For all analyses, a level of $5 \%$ or lower was considered significant. Data were analyzed with non-parametric tests using the SPSS version 20 (IBM, Armonk, NY).

\section{RESULTS}

In Spain, 22 universities offer the pharmacy degree required to practice (Grado en Farmacia), of which 13 are public and 9 are private institutions. In these 22 pharmacy degrees, 1261 courses are offered, of which $74.7 \%$ correspond to mandatory courses $(n=942)$. The distribution of courses per institution is presented in Table 2. The percentage of mandatory courses differs from $79.4 \%$ in private universities to $71.3 \%$ in public institutions $(p=0.001)$.

All 22 institutions present on their website the curricular plan as a list of courses and their distribution throughout the degree, as well as the number of corresponding ECTS. The course syllabus is available on the website for $95.5 \%$ of the courses $(n=1204)$. Eleven universities $(50 \%)$ have all syllabi available on the website. Of note are the URL, UAX and UCAM, with only $73.2 \%$, $82.1 \%$ and $83.7 \%$ of the course syllabi available on the website, respectively. From the 942 mandatory courses, 47 correspond to the internship period outside the university or to the final dissertation and the other 14 do not have the syllabus available at the website. Thus, a total of 881 courses were analyzed: 394 (44.7\%) from private universities and 487 (55.3\%) from public universities.

Only $507(57.5 \%)$ courses were paired to a CIN/2137/2008 general competency. In five universities, all courses were paired to a general competency, while two universities had no courses paired to general competencies. Table 3 shows the distribution per university of general competencies assigned to courses. Very different allocation patterns are observed, ranging from universities assigning a median of 1 general competency per course to two universities assigning 15 (out of 15) competencies per course. Table 4 shows the number of courses assigned to each of the 15 general competencies per university. Overall, general competencies were unevenly allocated to courses, ranging from 32.6\% of the courses allocated to competency \#15 (self-learning), to only $11.7 \%$ of courses allocated to competency \#8 (clinical and social pharmacy activities).

Online Appendix 1 shows the number of courses peruniversity that assigned specific competencies presented by the 7 areas of knowledge stated in the CIN/2137/2008 Ministerial order. The pairing of specific competencies greatly varies from university to university. Some universities assign the same specific competency to several different courses (e.g., the ULL assigns SC1 to 10 courses) while others assign that competency to only 1 course (e.g., the UV attributes SC1 to 1 course). Other universities with general competencies assigned to each course do not have specific competencies assigned (e.g., UB). Interestingly, although we have excluded from the analysis the courses associated with the internship and the final dissertation, some mandatory courses of several universities allocate specific competencies to the internship and the final dissertation area.

Of the 881 courses, $560(63.6 \%)$ could be objectively associated with one of the 7 areas of knowledge considered in the CIN/2137/2008 Ministerial order, 


\section{Table 2: Distribution of Courses per University ( $n=1261)$.}

\begin{tabular}{|c|c|c|c|}
\hline Universities & Mandatory Courses (\%) & Elective Courses (\%) & $\begin{array}{l}\text { Total Number of } \\
\text { Courses Offered }\end{array}$ \\
\hline University of Pais Vasco (UPV) & $74.5(n=35)$ & $25.5(n=12)$ & 47 \\
\hline University of Alcala de Henares (UAH) & $61.5(n=32)$ & $38.5(n=20)$ & 52 \\
\hline University of Barcelona (UB) & $53.7(n=44)$ & $46.3(n=38)$ & 82 \\
\hline University of Santiago de Compostela (USC) & $71.9(n=46)$ & $28.1(n=18)$ & 64 \\
\hline University of Granada (UGR) & $69.5(n=41)$ & $30.5(n=18)$ & 59 \\
\hline University of Salamanca (USAL) & $65.1(n=41)$ & $34.9(n=22)$ & 63 \\
\hline University Complutense Madrid (UCM) & $62.3(n=38)$ & $37.7(n=23)$ & 61 \\
\hline University Alfonso X El Sabio (UAX) & $73.2(n=41)$ & $26.8(n=15)$ & 56 \\
\hline University of Navarra (UN) & $70.0(n=49)$ & $30.0(n=21)$ & 70 \\
\hline University of La Laguna (ULL) & $86(n=37)$ & $14(n=6)$ & 43 \\
\hline University Francisco de Vitoria (UFV) & $83.6(n=46)$ & $16.4(n=9)$ & 55 \\
\hline University Cardenal Herrera CEU (UCH) & $92.3(n=48)$ & $7.7(n=4)$ & 52 \\
\hline University of Sevilla (US) & $73.2(n=41)$ & $26.8(n=15)$ & 56 \\
\hline University San Pablo CEU (USP) & $67.6(n=48)$ & $32.4(n=23)$ & 71 \\
\hline University Miguel Hernández (UMH) & $88.9(n=48)$ & $11.1(n=6)$ & 54 \\
\hline University of Valencia (UV) & $70.9(n=39)$ & $29.1(n=16)$ & 55 \\
\hline University San Jorge (USJ) & $83.3(n=45)$ & $16.7(n=9)$ & 54 \\
\hline University of Murcia (UM) & $76.7(n=33)$ & $23.3(n=10)$ & 43 \\
\hline University Europea de Madrid (UEM) & $91.1(n=51)$ & $8.9(n=5)$ & 56 \\
\hline University of Castilla-La Mancha (UCLM) & $89.6(n=43)$ & $10.4(n=5)$ & 48 \\
\hline University Católica de Murcia (UCAM) & $91.8(n=45)$ & $8.2(n=4)$ & 49 \\
\hline University Ramán Llul (URL) & $71.8(n=51)$ & $28.2(n=20)$ & 71 \\
\hline
\end{tabular}

\begin{tabular}{|c|c|c|c|c|c|c|}
\hline & \multirow[b]{2}{*}{ Courses w/o GC } & \multirow[b]{2}{*}{ Courses w/ GC (\%) } & \multicolumn{4}{|c|}{ Number of GC assigned per course } \\
\hline & & & Mean & SD & Median & IQR (Q3 : Q1) \\
\hline UPV & 25 & $8(24.2 \%)$ & 2,88 & 2,03 & 2 & $2: 4.25$ \\
\hline $\mathrm{UAH}$ & 23 & $7(23.3 \%)$ & 4 & 2 & 4 & $2: 5$ \\
\hline UB & 8 & $34(81.0 \%)$ & 2,74 & 1,19 & 2 & $1: 4$ \\
\hline USC & 21 & $23(52.3 \%)$ & 3,61 & 2,11 & 3 & $2: 5$ \\
\hline UGR & 3 & $35(92.1 \%)$ & 4,54 & 2,42 & 4 & $3: 7$ \\
\hline USAL & 35 & $3(7.9 \%)$ & 2,67 & 0,58 & 3 & $3: 3$ \\
\hline UCM & 1 & $35(97.2 \%)$ & 12,46 & 4,78 & 15 & $15: 15$ \\
\hline UAX & 35 & $4(10.3 \%)$ & 5,25 & 6,55 & 2,5 & $1.25: 12$ \\
\hline UN & 11 & $35(76.1 \%)$ & 2,37 & 1,4 & 2 & $1: 3$ \\
\hline ULL & 0 & $35(100 \%)$ & 3,2 & 1,64 & 3 & $2: 4$ \\
\hline UFV & 0 & $44(100 \%)$ & 3,75 & 2,65 & 3 & $2: 6$ \\
\hline $\mathrm{UCH}$ & 28 & $18(39.1 \%)$ & 3,39 & 2,45 & 3,5 & $1: 5$ \\
\hline US & 31 & $8(20.5 \%)$ & 3,63 & 2,33 & 2,5 & $2: 6$ \\
\hline USP & 46 & $0(0.0 \%)$ & - & - & - & - \\
\hline UMH & 43 & $0(0.0 \%)$ & - & - & - & - \\
\hline UV & 15 & $22(59.5 \%)$ & 2,36 & 1,97 & 1,5 & $1: 4$ \\
\hline USJ & 0 & $43(100 \%)$ & 2,19 & 1,22 & 2 & $1: 3$ \\
\hline UM & 0 & $31(100 \%)$ & 7,1 & 4,18 & 6 & $3: 12$ \\
\hline UEM & 40 & $3(7.0 \%)$ & 1 & 0 & 1 & $1: 1$ \\
\hline UCLM & 1 & $40(97.6 \%)$ & 12,78 & 3,72 & 15 & $10: 15$ \\
\hline UCAM & 8 & $31(79.5 \%)$ & 5,23 & 4,21 & 4 & $2: 6$ \\
\hline URL & 0 & $48(100 \%)$ & 2,69 & 1,55 & 2 & $2: 3$ \\
\hline Total & 374 & 507 & & & & \\
\hline
\end{tabular}




\begin{tabular}{|c|c|c|c|c|c|c|c|c|c|c|c|c|c|c|c|c|c|c|c|c|c|c|c|}
\hline ¿্ঠ & 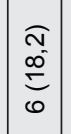 & $\begin{array}{l}0 \\
0 \\
\stackrel{0}{c} \\
m\end{array}$ & \begin{tabular}{|l|}
$\widehat{\sigma}$ \\
$\mathfrak{y}$ \\
$\mathbb{y}$ \\
$\stackrel{\infty}{\leftarrow}$
\end{tabular} & 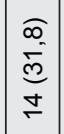 & 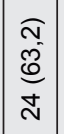 & $\begin{array}{l}\bar{m} \\
\tilde{e} \\
\stackrel{0}{N}\end{array}$ & $\begin{array}{l}\widehat{N} \\
\hat{N} \\
0 \\
\omega \\
\infty \\
0\end{array}$ & $\begin{array}{c}\underset{\sigma}{\mathfrak{d}} \\
\stackrel{-}{-}\end{array}$ & 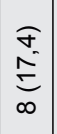 & 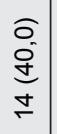 & 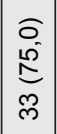 & 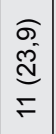 & 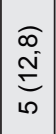 & & & 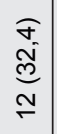 & \begin{tabular}{|l|}
$\widehat{\widehat{c}}$ \\
$\overline{0}$ \\
$\underline{\underline{p}}$ \\
$\underline{\underline{c}}$
\end{tabular} & 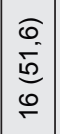 & 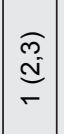 & 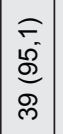 & 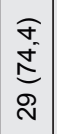 & $\begin{array}{l}\bar{m} \\
\hat{e} \\
\text { m }\end{array}$ & \\
\hline $\bar{u}$ & $\begin{array}{c}\stackrel{\partial}{0} \\
\stackrel{\Gamma}{\sigma} \\
\ulcorner\end{array}$ & $\begin{array}{c}\tilde{m} \\
\stackrel{n}{-} \\
-\end{array}$ & $\mid \begin{array}{c}\mathfrak{f} \\
\mathfrak{d} \\
r\end{array}$ & 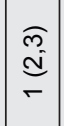 & $\begin{array}{l}\widehat{N} \\
\tilde{\omega} \\
N \\
\tilde{N}\end{array}$ & ' & $\begin{array}{l}\tilde{E} \\
\infty \\
\infty \\
\bar{m}\end{array}$ & $\begin{array}{c}\underset{\mathfrak{v}}{\mathfrak{v}} \\
-\end{array}$ & $\begin{array}{l}\widetilde{R} \\
\infty \\
\sigma \\
\sigma\end{array}$ & $\begin{array}{l}\underset{\sigma}{\mathcal{I}} \\
-\end{array}$ & $\begin{array}{c}\widetilde{E} \\
\vec{O} \\
\forall\end{array}$ & $\begin{array}{l}\underset{\mathfrak{v}}{\mathcal{d}} \\
-\end{array}$ & $\begin{array}{l}\hat{E} \\
\bar{E} \\
m\end{array}$ & & ' & $\begin{array}{l}\underset{\mathfrak{d}}{\mathbb{N}} \\
-\end{array}$ & $\begin{array}{l}\tilde{R} \\
\dot{j} \\
\sim\end{array}$ & 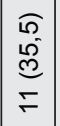 & & \begin{tabular}{|c|}
$\widehat{N}$ \\
$\tilde{d}$ \\
\\
0 \\
$\tilde{d}$
\end{tabular} & 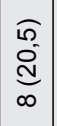 & $\begin{array}{l}\underset{\sigma}{\sigma} \\
\stackrel{+}{\Xi} \\
r\end{array}$ & \\
\hline U্ঠ & $\left|\begin{array}{c}\mathfrak{N} \\
\multirow{d}{d}{} \\
\infty \\
\infty\end{array}\right|$ & 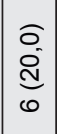 & 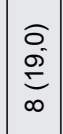 & \begin{tabular}{|l|}
$\tilde{\sigma}$ \\
6 \\
0 \\
0 \\
0 \\
$\underline{0}$
\end{tabular} & $\begin{array}{l}\sigma \\
0 \\
b \\
o \\
\sigma \\
\sigma\end{array}$ & 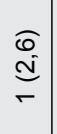 & $\begin{array}{l}\widehat{\sigma} \\
\infty \\
\infty \\
\tilde{\omega} \\
\tilde{\infty}\end{array}$ & $\begin{array}{c}\underset{b}{b} \\
\sim\end{array}$ & $\begin{array}{c}\sigma \\
0 \\
0 \\
0 \\
0\end{array}$ & 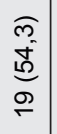 & $\begin{array}{l}\tilde{N} \\
\hat{j} \\
\bar{N} \\
\bar{N}\end{array}$ & $\begin{array}{l}0 \\
0 \\
0 \\
0 \\
0\end{array}$ & 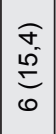 & & ' & $\begin{array}{l}\widehat{0} \\
\stackrel{-}{\underline{d}} \\
\infty\end{array}$ & $\mid \begin{array}{l}\widehat{\sigma} \\
\stackrel{D}{D} \\
\sigma\end{array}$ & 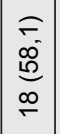 & & 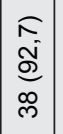 & $\begin{array}{l}\widehat{c} \\
\stackrel{-}{5} \\
\stackrel{5}{n} \\
\stackrel{n}{n}\end{array}$ & $\begin{array}{l}\widehat{E} \\
\stackrel{0}{0} \\
\stackrel{0}{0} \\
\infty\end{array}$ & \\
\hline O & $\begin{array}{c}\hat{E} \\
\stackrel{e}{v} \\
v\end{array}$ & $\mid \begin{array}{c}\hat{n} \\
\tilde{c} \\
-\end{array}$ & $\begin{array}{l}\tilde{E} \\
\overline{0} \\
m\end{array}$ & $\mid \begin{array}{c}\bar{\sigma} \\
\sigma \\
\sigma\end{array}$ & 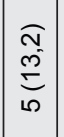 & ' & $\begin{array}{l}\widehat{\infty} \\
\hat{N} \\
\stackrel{\infty}{N} \\
\stackrel{N}{N}\end{array}$ & $\mid \begin{array}{c}0 \\
\mathfrak{d} \\
-\end{array}$ & $\begin{array}{l}\tilde{m} \\
\dot{ \pm} \\
N\end{array}$ & 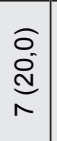 & $\begin{array}{l}0 \\
0 \\
e \\
m\end{array}$ & $\begin{array}{l}\underset{\mathbb{V}}{\mathfrak{d}} \\
-\end{array}$ & $\begin{array}{c}0 \\
\mathfrak{\sigma} \\
\mathfrak{d} \\
-\end{array}$ & ' & ' & $\begin{array}{c}\overparen{ } \\
\infty \\
\infty \\
m\end{array}$ & $\begin{array}{l}\mathbb{R} \\
\dot{J} \\
N\end{array}$ & 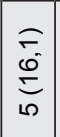 & & \begin{tabular}{|l|}
$\widehat{\alpha}$ \\
$\widehat{\infty}$ \\
$\infty$ \\
$o$ \\
$e$ \\
\end{tabular} & 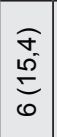 & $\begin{array}{l}\widehat{m} \\
\infty \\
\sigma \\
\sigma\end{array}$ & \\
\hline 文 & & ' & 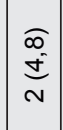 & \begin{tabular}{|l|}
$\hat{\alpha}$ \\
$\hat{0}$ \\
$\tilde{m}$
\end{tabular} & $\left|\begin{array}{c}0 \\
\stackrel{d}{d} \\
-\end{array}\right|$ & ' & $\begin{array}{l}0 \\
i \stackrel{0}{s} \\
\stackrel{N}{N}\end{array}$ & 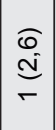 & $\begin{array}{l}\underset{\mathfrak{d}}{\mathrm{d}} \\
-\end{array}$ & 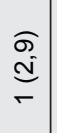 & $\begin{array}{c}\widehat{\mathfrak{m}} \\
\stackrel{\mathrm{v}}{-} \\
-\end{array}$ & & $\mid \begin{array}{c}\bar{\sigma} \\
\frac{6}{N}\end{array}$ & & ' & & $\begin{array}{l}\tilde{r} \\
\tilde{j} \\
\sim\end{array}$ & 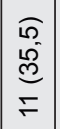 & 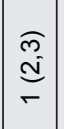 & 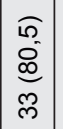 & 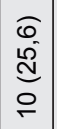 & 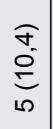 & \\
\hline 엉 & $\mid \begin{array}{c}\widehat{\partial} \\
\tilde{\sigma} \\
-\end{array}$ & $\begin{array}{l}\bar{m} \\
\stackrel{n}{c} \\
-\end{array}$ & 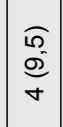 & 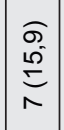 & 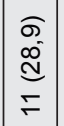 & ' & 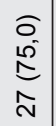 & $\begin{array}{c}\underset{\sigma}{d} \\
\stackrel{\mathfrak{v}}{-} \\
-\end{array}$ & 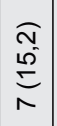 & $\begin{array}{l}\mathbb{E} \\
\stackrel{\mathfrak{d}}{0} \\
\sigma \\
\sigma\end{array}$ & 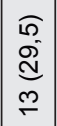 & 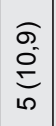 & ' & ' & ' & $\begin{array}{l}\widehat{f} \\
\stackrel{5}{b} \\
\sim\end{array}$ & 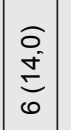 & 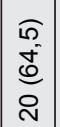 & & 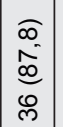 & 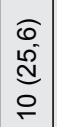 & 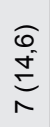 & \\
\hline ¿ & $\begin{array}{c}\widehat{\sigma} \\
\tilde{m}^{-} \\
\sigma\end{array}$ & 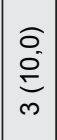 & 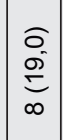 & \begin{tabular}{|c|}
$\widehat{N}$ \\
$\infty$ \\
$\infty$ \\
$\infty$ \\
$\infty$
\end{tabular} & 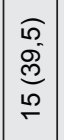 & $\begin{array}{l}\widehat{\sigma} \\
\mathfrak{d} \\
-\end{array}$ & $\begin{array}{l}\widehat{\infty} \\
\hat{N} \\
\stackrel{\infty}{N} \\
\stackrel{N}{N}\end{array}$ & $\mid \begin{array}{c}0 \\
\mathfrak{d} \\
-\end{array}$ & $\begin{array}{l}\sigma \\
0 \\
0 \\
0 \\
0\end{array}$ & 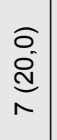 & 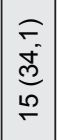 & $\begin{array}{l}\underset{f}{N} \\
\stackrel{N}{5} \\
\infty \\
\infty\end{array}$ & $\mid \begin{array}{c}0 \\
\stackrel{0}{d} \\
-\end{array}$ & ' & ' & $\begin{array}{l}\widehat{0} \\
\stackrel{0}{0} \\
\stackrel{0}{\sigma} \\
\vec{\sigma}\end{array}$ & $\mid$\begin{tabular}{c|}
6 \\
$\infty$ \\
$\infty$ \\
$\infty$ \\
$\infty$ \\
0
\end{tabular} & 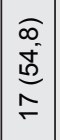 & & 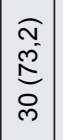 & 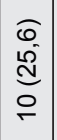 & $\begin{array}{l}\underset{0}{0} \\
\stackrel{+}{5}\end{array}$ & \\
\hline u & & ' & $\mid \begin{array}{c}\tilde{\sigma} \\
\mathfrak{d} \\
-\end{array}$ & 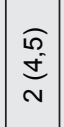 & 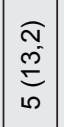 & ' & 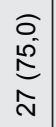 & 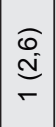 & 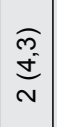 & $\begin{array}{l}\underset{\mathfrak{d}}{\mathcal{N}} \\
-\end{array}$ & $\begin{array}{c}\vec{\sigma} \\
\bar{O} \\
\sigma\end{array}$ & $\begin{array}{l}\tilde{N} \\
\underset{\sim}{ \pm} \\
\sim\end{array}$ & 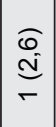 & & & 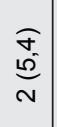 & $\mid \begin{array}{l}\tilde{m} \\
\tilde{\sigma} \\
\sigma \\
\sigma\end{array}$ & $\begin{array}{c}\hat{\tilde{n}} \\
\tilde{N} \\
\tilde{d} \\
\stackrel{0}{\circ}\end{array}$ & 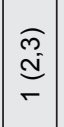 & $\mid \begin{array}{l}\widehat{N} \\
\tilde{N} \\
\\
0 \\
\tilde{d}\end{array}$ & $\begin{array}{c}1 \\
0 \\
2 \\
\infty \\
\infty\end{array}$ & $\begin{array}{l}\underset{v}{N} \\
\underset{N}{ \pm}\end{array}$ & \\
\hline O্ & ' & $\begin{array}{l}E \\
e \\
v \\
N\end{array}$ & \begin{tabular}{|l|} 
\\
0 \\
0 \\
$\sigma$ \\
$\sigma$
\end{tabular} & $\begin{array}{l}\mathbb{1} \\
\stackrel{0}{ \pm} \\
\sim\end{array}$ & 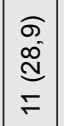 & ' & $\begin{array}{l}\hat{\alpha} \\
\hat{N}\end{array}$ & 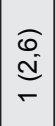 & $\begin{array}{l}1 \\
0 \\
0 \\
m\end{array}$ & 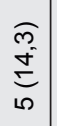 & 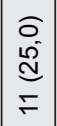 & $\begin{array}{l}10 \\
0 \\
0 \\
m\end{array}$ & $\begin{array}{c}\underset{\sigma}{\sigma} \\
\stackrel{v}{-} \\
-\end{array}$ & ' & ' & $\begin{array}{l}\underset{\mathfrak{d}}{\mathrm{g}} \\
-\end{array}$ & 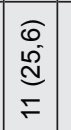 & 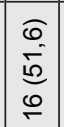 & & \begin{tabular}{|c|}
$\underline{\alpha}$ \\
$\hat{\alpha}$ \\
$\infty$ \\
0 \\
0 \\
\end{tabular} & $\begin{array}{l}\sigma \\
\stackrel{\sigma}{N} \\
\stackrel{N}{N}\end{array}$ & $\underset{0}{\stackrel{10}{\mathbb{N}}}$ & \\
\hline ن & & $\begin{array}{l}\tilde{E} \\
e \\
v\end{array}$ & 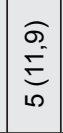 & \begin{tabular}{|l|}
$\widehat{o}$ \\
$e$ \\
$e$ \\
$m$
\end{tabular} & $\begin{array}{l}\widehat{\widehat{Q}} \\
\stackrel{\vec{g}}{\mathrm{~N}} \\
\stackrel{\sim}{\sim}\end{array}$ & ' & $\begin{array}{l}\widehat{\infty} \\
\stackrel{N}{N} \\
\infty \\
\sim \\
\sim\end{array}$ & $\begin{array}{c}\widetilde{c} \\
\stackrel{5}{n} \\
N\end{array}$ & $\begin{array}{l}10 \\
0 \\
0\end{array}$ & 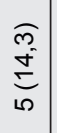 & 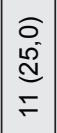 & $\begin{array}{l}10 \\
0 \\
e \\
m\end{array}$ & $\begin{array}{c}\bar{\sigma} \\
\frac{60}{N} \\
\sim\end{array}$ & ' & ' & $\begin{array}{l}\widehat{\infty} \\
\stackrel{0}{0} \\
= \\
\sigma\end{array}$ & $\begin{array}{l}\tilde{n} \\
0 \\
0 \\
\\
r\end{array}$ & $\mid \begin{array}{l}0 \\
0- \\
\underline{0} \\
0 \\
0\end{array}$ & & 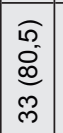 & $\begin{array}{l}\sigma \\
\stackrel{\sigma}{N} \\
\stackrel{N}{n}\end{array}$ & 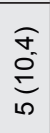 & \\
\hline us & $\stackrel{\overbrace{}}{e}$ & 1 & \begin{tabular}{|l|}
0 \\
0 \\
0 \\
$\sigma$ \\
$\sigma$
\end{tabular} & $\mid \begin{array}{c}\widehat{\tilde{n}} \\
\stackrel{\mathrm{v}}{\mathbf{v}}\end{array}$ & $\begin{array}{c}\stackrel{\vec{c}}{\vec{c}} \\
\infty \\
\infty\end{array}$ & $\begin{array}{l}\underset{\sigma}{d} \\
\stackrel{v}{-}\end{array}$ & $\begin{array}{l}\widehat{\alpha} \\
\hat{N} \\
\stackrel{0}{\infty} \\
\stackrel{0}{N}\end{array}$ & $\mid \begin{array}{c}\sigma \\
\stackrel{d}{-} \\
-\end{array}$ & 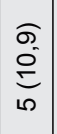 & 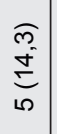 & \begin{tabular}{|c|c}
0 \\
$\stackrel{0}{0}$ \\
$\stackrel{0}{0}$ \\
0
\end{tabular} & $\left|\begin{array}{c}\hat{\sigma} \\
\tilde{d} \\
\text { v }\end{array}\right|$ & ' & ' & ' & $\begin{array}{l}\hat{\sigma} \\
\stackrel{d}{\sigma}\end{array}$ & $\mid \begin{array}{l}\tilde{m} \\
\tilde{0} \\
\sigma\end{array}$ & 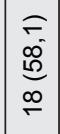 & & 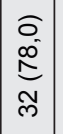 & 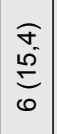 & $\begin{array}{l}\widehat{\sigma} \\
\alpha^{0} \\
\sigma\end{array}$ & \\
\hline ن્ & ' & ' & $\begin{array}{l}\tilde{L} \\
0 \\
\sigma \\
\sigma\end{array}$ & \begin{tabular}{|l|}
$\hat{\alpha}$ \\
$\hat{e}$ \\
$m$
\end{tabular} & 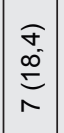 & 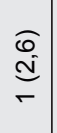 & $\begin{array}{l}\widehat{\tilde{m}} \\
\tilde{j} \\
\infty \\
0 \\
\tilde{m}\end{array}$ & $\begin{array}{c}0 \\
\mathfrak{d} \\
- \\
-\end{array}$ & $\begin{array}{l}\mathbb{2} \\
\stackrel{-}{\mathrm{d}} \\
\stackrel{0}{\circ}\end{array}$ & $\begin{array}{l}\underset{\sigma}{\mathbb{N}} \\
\underset{\infty}{\mathbb{N}}\end{array}$ & $\begin{array}{c}\widehat{N} \\
\infty \\
\stackrel{\infty}{C} \\
\infty\end{array}$ & $\begin{array}{l}0 \\
0 \\
0 \\
0 \\
\text { ம }\end{array}$ & $\begin{array}{c}\widetilde{c} \\
\vec{b} \\
N\end{array}$ & & & $\begin{array}{l}\hat{\mathbb{E}} \\
\stackrel{\mathfrak{d}}{-}\end{array}$ & $\begin{array}{l}\tilde{E} \\
\mathbb{J} \\
N\end{array}$ & $\mid \begin{array}{c}\mathbb{N} \\
\infty \\
0 \\
\stackrel{0}{\simeq} \\
\cong\end{array}$ & & 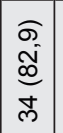 & $\begin{array}{c}1 \\
0 \\
2 \\
\infty \\
\infty\end{array}$ & 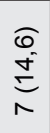 & \\
\hline ( & $\begin{array}{l}\widehat{\sigma} \\
\tilde{m}^{-} \\
\end{array}$ & $\begin{array}{l}0 \\
0 \\
0 \\
0 \\
0\end{array}$ & \begin{tabular}{|l|}
$\widehat{o}$ \\
$\bar{m}$ \\
$\underline{m}$ \\
$\underline{m}$
\end{tabular} & 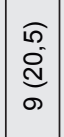 & 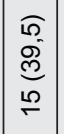 & 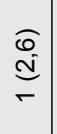 & $\begin{array}{l}\widehat{\tilde{m}} \\
\tilde{\infty} \\
\infty \\
0 \\
\tilde{m}\end{array}$ & 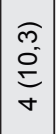 & $\begin{array}{c}\sigma \\
0 \\
0 \\
0 \\
0\end{array}$ & 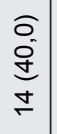 & 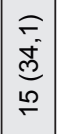 & 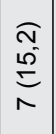 & $\begin{array}{c}\widetilde{ } \\
\omega^{0} \\
\sim\end{array}$ & ' & ' & $\begin{array}{l}\hat{m} \\
\mathfrak{d} \\
\tilde{d} \\
\sigma\end{array}$ & 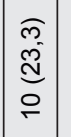 & \begin{tabular}{|l}
$\widehat{\mathfrak{N}}$ \\
$\mathbb{J}$ \\
$\tilde{N}$ \\
$\widetilde{N}$
\end{tabular} & & \begin{tabular}{|c|}
$\hat{N}$ \\
$\hat{N}$ \\
$\tilde{o}$ \\
$\infty$ \\
$\tilde{e}$
\end{tabular} & $\begin{array}{c}1 \\
0 \\
0 \\
\infty \\
\infty\end{array}$ & $\begin{array}{l}\infty \\
0 \\
0 \\
\infty\end{array}$ & \\
\hline U & ' & $\begin{array}{l}\widehat{\rho} \\
\hat{\sigma} \\
\underline{n}\end{array}$ & \begin{tabular}{|c|}
$\widehat{m}$ \\
$\stackrel{0}{5}$ \\
0 \\
0
\end{tabular} & 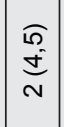 & $\begin{array}{l}\overrightarrow{-} \\
\bar{d} \\
\infty \\
\infty\end{array}$ & ' & $\begin{array}{l}\widehat{a} \\
\dot{b} \\
\hat{N} \\
\hat{N}\end{array}$ & 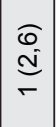 & 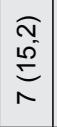 & $\begin{array}{l}\tilde{E} \\
\stackrel{1}{E} \\
0\end{array}$ & 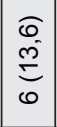 & $\begin{array}{l}10 \\
0 \\
0 \\
m\end{array}$ & $\begin{array}{l}0 \\
\mathfrak{d} \\
- \\
-\end{array}$ & , & ' & $\begin{array}{l}\vec{\sigma} \\
\vec{b} \\
\sim\end{array}$ & 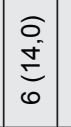 & 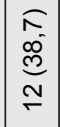 & & $\mid \begin{array}{c}\hat{N} \\
\tilde{N} \\
\tilde{D} \\
0 \\
\tilde{n}\end{array}$ & $\begin{array}{l}\overrightarrow{\tilde{d}} \\
\stackrel{d}{d} \\
\sigma\end{array}$ & $\begin{array}{l}\widehat{\infty} \\
\text { D. } \\
0 \\
0\end{array}$ & \\
\hline ن & 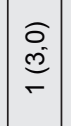 & $\begin{array}{l}\tilde{N} \\
\hat{e} \\
\sim\end{array}$ & 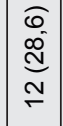 & \begin{tabular}{|c|}
$\widehat{a}$ \\
$\infty$ \\
$\underbrace{\infty}_{\infty}$ \\
$\infty$
\end{tabular} & $\begin{array}{c}\tilde{z} \\
\tilde{y} \\
\stackrel{y}{0}\end{array}$ & $\begin{array}{l}\underset{\sigma}{\stackrel{d}{d}} \\
-\end{array}$ & 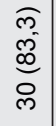 & $\begin{array}{c}\underset{c}{\sigma} \\
\sim \\
N\end{array}$ & 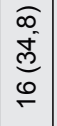 & $\begin{array}{l}\widehat{\sigma} \\
\infty \\
\stackrel{0}{0} \\
\stackrel{0}{0}\end{array}$ & 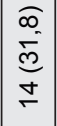 & $\begin{array}{c}\sigma \\
0 \\
0 \\
\\
0\end{array}$ & 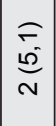 & , & ' & $\begin{array}{l}\tilde{f} \\
b \\
v \\
v\end{array}$ & 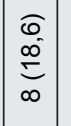 & 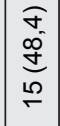 & & 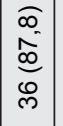 & 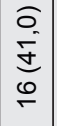 & & \\
\hline 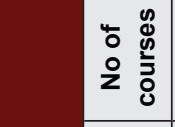 & m & లి & ₹ & 棌 & $\stackrel{\infty}{\infty}$ & $\stackrel{\infty}{\infty}$ & $\stackrel{\infty}{\infty}$ & 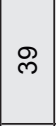 & $\mathscr{q}$ & 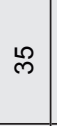 & $\forall$ & $q$ & & $\mathscr{q}$ & $\stackrel{?}{q}$ & $\hat{m}$ & $\stackrel{m}{q}$ & $\bar{m}$ & F & $\subsetneq$ & 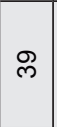 & $\stackrel{\infty}{+}$ & $\infty$ \\
\hline ¿̇ & 긱 & $S$ & $\stackrel{m}{د}$ & כ) & תי & 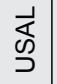 & $\sum_{\mathrm{J}}$ & 走 & z & $\vec{J}$ & 站 & $\begin{array}{l}\mathrm{I} \\
\mathrm{J}\end{array}$ & I & $\frac{n}{0}$ & $\sum_{J}^{T}$ & 3 & $\overrightarrow{\mathcal{D}}$ & 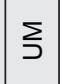 & 站 & 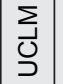 & $\begin{array}{l}\sum_{0} \\
\text { J }\end{array}$ & 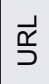 & \\
\hline
\end{tabular}


with only $16(1.8 \%)$ classified as non-specific (because of a tie). The distribution of these 560 courses per areas of knowledge resulted in $133(23.8 \%)$ in chemistry, $38(6.8 \%)$ in physics and mathematics, $92(16.4 \%)$ in biology, $73(13.0 \%)$ in pharmaceutical technology, 166 $(29.6 \%)$ in medicine and pharmacology, $57(10.2 \%)$ in legislation and social pharmacy and 1 course $(0.2 \%)$ in the internship (not provided during the internship period).

Table 5 shows the distribution of the number of courses with each general competency allocated, grouped by the objectively classified area of knowledge. The difference between the total number of courses per general competency (Table 5) and the total number of courses with that competency assigned (Table 4) is due to the number of courses with no specific competencies assigned, which required us to objectively classify the courses into the CIN/2137/2008 areas of knowledge. Thus, Table 5 identifies the proportion of the courses from each area of knowledge that provides each of the general competencies. For instance, $40.4 \%$ of the courses paired with general competency \#1 (analyze and produce medicines) are from the chemistry area. Other general competencies present a wider range of pairings of courses and areas of knowledge. For instance, general competency \#8 (clinical and social pharmacy activities) was assigned to $51.5 \%$ of courses from medicine and pharmacology, 20.6\% from chemistry, $10.3 \%$ from pharmaceutical technology and only $6.2 \%$ from legislation and social pharmacy. General competency \#13 (communication and information skills) was assigned to $36.8 \%$ of courses from medicine and pharmacology, $17.3 \%$ from chemistry, $15.9 \%$ from biology, 15.5\% from pharmaceutical technology and only $7.7 \%$ from legislation and social pharmacy.

\section{DISCUSSION}

We found that the Spanish competency framework for undergraduate pharmacy education, although legally enforced since 2008 by the CIN/2137/2008 Ministerial order, is not properly used. Competencies are frequently mismatched, with no real alignment between them and the courses' educational content. Despite the legal obligation, only five out of the 22 universities providing the pharmacy degree in Spain have all their courses paired with any of the general competencies of the Spanish competency framework and two universities have none of their courses paired with any of the general competencies.

The number of competences assigned by course gives also demonstrates very different alignment patterns

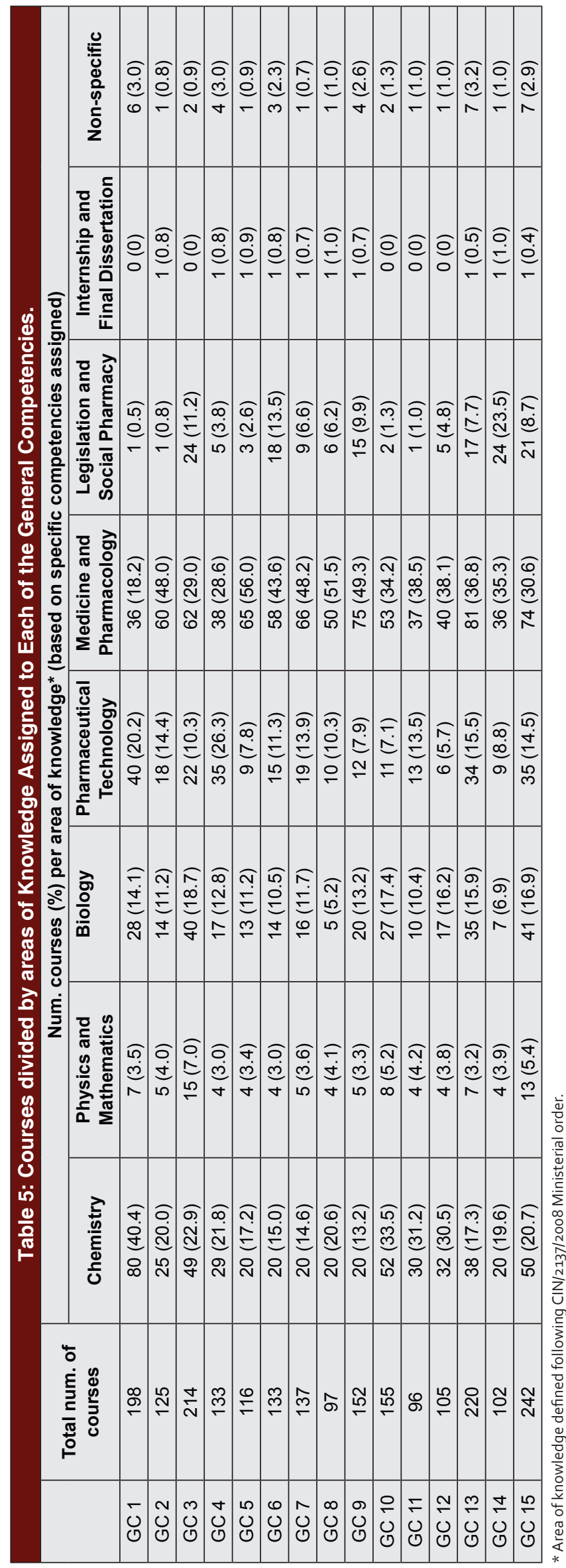


among Spanish colleges of pharmacy. While some universities pair each course to a median of around two competencies, some others align virtually all their courses to all the 15 general competencies. This excessive pairing may be another way of misusing a competency framework, pretending that each course provides many different competencies, such as \#1 (analyze and produce medicines) and \#8 (clinical and social pharmacy activities). The use of a competency framework should support the construction and implementation of a competencybased pharmacy education. ${ }^{22,23}$ Therefore, the curriculum must be designed to ensure a perfect alignment between educational outcomes, educational contents, teaching and learning methods, assessment strategies and educational environment. ${ }^{1,4,22,23}$

Competence mismatching in Spain is most evident in the patient-focused competencies. Among the five universities that have all their courses paired with any general competency (Table 4), a variability in the assignment of general competency \#7 (drug-related problems and pharmacovigilance activities) exists, ranging from $13 \%$ of the URL courses to $52 \%$ of the UM courses. In the case of general competency \#8 (clinical and social pharmacy activities), its assignment varies from $3 \%$ of the ULL courses to $32 \%$ of the UM courses. The results of our study show that UM assigned general competency \#7 four times more often than URL and assigned general competency \#8 ten times more often than ULL. This finding could lead us to think that the pharmacy degree in UM is much more patient-focused than in the other two universities, but an in-depth analysis of their syllabi shows very similar courses regarding the declared educational contents. Although there is a great variability in the assignment of competencies, overall, the Spanish universities assign general competency \#7 (drug-related problems and pharmacovigilance activities) and general competency \#8 (clinical and social pharmacy activities) to only $17 \%$ and $12 \%$ of the courses, respectively. The low assignment rate of these two competencies reinforces the results of a previous comparison between the U.S. and European curricula, concluding that U.S. curricula were more clinically oriented. ${ }^{24}$

Spanish colleges of pharmacy competency alignments demonstrate an attempt to simulate a more clinically oriented pharmacy curriculum. Several of the patientfocused general competencies are provided by courses paired with very different profiles of specific competencies. Table 5 shows that $44 \%$ of the courses that have assigned general competency \#7 (drug-related problems and pharmacovigilance activities) and 40\% of the courses that have assigned general competency \#8 (clinical and social pharmacy activities) belong to the areas of knowledge of chemistry, physics and mathematics, biology and pharmaceutical technology. Allocating courses to an area of knowledge based only on the name of the course may mislead curriculum analysis, as has already occurred with the 2011 PHARMINE report. ${ }^{25}$ Spanish universities should strive to make a correct alignment between the competencies from the Spanish competency framework and the educational contents taught in each class and not merely creating general pairings based on the courses' names. The US-based Accreditation Council for Pharmacy Education (ACPE) standards and guidelines argue that "the curriculum must define the expected outcomes and be developed, with attention to sequencing and integration of content and the selection of teaching and learning methods and assessments". ${ }^{26}$

Following this rationale, the competencies that are intended to be achieved by the students should be paired with educational content (the description of course content, including the sequence of topics and readings; the learning activities/assignments; and the time spent to reach each competency) and the assessment methods. It is important to bear in mind that the learning activities are used to develop each competency and the assessment strategies are used to evaluate if the student achieved each competency. ${ }^{5}$ Additionally, competencies should be aggregated into entrust able professional activities (EPAs): "units of professional practice, defined as tasks or responsibilities that trainees are entrusted to perform unsupervised once they have attained specific sufficient competence". ${ }^{27}$ Although EPAs should not be considered as an alternative to design curricula, ${ }^{27}$ competency assignment should also be analyzed from the EPAs perspective. At the end of the day, courses provide competencies and competencies support EPAs. A complete curriculum analysis should be able to map all this alignment, especially in core competencies. ${ }^{28}$ And different curricula should demonstrate their different orientations by comparing their coverage of specific sets of EPAs. ${ }^{29}$ With these modifications, one should be able to map the reasons why a curriculum should include a given course and what competencies and activities are the courses preparing the students for their professional practice. Educational and professional regulatory bodies should work on a common database to pair educational contents with competencies and professional activities.

\section{Limitations of the study}

One potential limitation of our study is that we excluded elective courses and courses related to the internship period or final dissertation. Exclusion of elective courses is based on the idea that mandatory courses are those 
common to any entry-level graduated and constitute the core contents taught to every pharmacist. The internship period was not considered for this study because, for the vast of the Spanish students, this period consists of a practice placement with no university-based educational contents.

\section{CONCLUSION}

Despite the use of the Spanish competency framework, a legal mandate enforced by the CIN/2137/2008 Ministerial order, its quantitative implementation is not complete (i.e., two universities do not assign any general competencies) and very different pairing patterns were found among universities. The quality of competency assignment is poor, with courses objectively assigned to basic science areas (i.e., chemistry, physics and mathematics, biology and pharmaceutical technology) providing high percentages of practical and patientfocused competencies. In the future, competencies should be paired in the syllabi to each of the course contents (lectures and labs) and not to the course descriptions.

\section{CONFLICT OF INTEREST}

The authors declare no conflict of interest.

\section{ABBREVIATIONS}

ACPE: Accreditation Council for Pharmacy Education; ECTS: European Credit Transfer and Accumulation System; EHEA: European Higher Education Area; EPA: Entrustable professional activities; FIP: International Pharmaceutical Federation; GC: General competency; SC: Specific competency; UAH: University of Alcala de Henares; UAX: University Alfonso X El Sabio; UB: University of Barcelona; UCAM: University Católica de Murcia; UCH: University Cardenal Herrera CEU; UCLM: University of Castilla-La Mancha; UCM: University Complutense Madrid; UEM: University Europea de Madrid; UFV: University Francisco de Vitoria; UGR: University of Granada; ULL: University of La Laguna; UM: University of Murcia; UMH: University Miguel Hernández; UN: University of Navarra; UNESCO: United Nations Educational, Scientific and Cultural Organization; UPV: University of Pais Vasco; URL: University Ramón Llul; US: University of Sevilla; USAL: University of Salamanca; USC: University of Santiago de Compostela; USJ: University San Jorge; USP: University San Pablo CEU; UV:
University of Valencia; WHO: World Health Organization.

\section{REFERENCES}

1. Santos WS. The Competency-Based Medical Curriculum. Rev Bras Educ Méd. 2011;35(1):86-92.

2. Sánchez-Pozo A. A Comparison of Competences for Healthcare Professions in Europe. Pharmacy. 2017;5(1):8.

3. Nash RE, Chalmers L, Brown N, Jackson S, Peterson G. An international review of the use of competency standards in undergraduate pharmacy education. Pharm Educ. 2015;15(1):131-41.

4. International Pharmaceutical Federation. Quality Assurance of Pharmacy Education: The FIP Global Framework. The Hague: FIP. 2014. Available from: https://www.fip.org/files/fip/PharmacyEducation/Quality_Assurance/ QA_Framework_2nd_Edition_online_version.pdf

5. Nunes-da-Cunha I, Fernandez-Llimos F. Educational contents for a patientcentred undergraduate pharmacy curriculum. Lisbon: CIPF. 2017. Available from: https://www.pharmacypractice.org/educational_contents/

6. International Pharmaceutical Federation. Pharmacy Education Taskforce. A Global Competency Framework for Services Provided by Pharmacy Workforce. The Hague. FIP. 2012.

7. Atkinson J. The Production of the PHAR-QA Competence Framework. Pharmacy. 2017;5(2):19.

8. The Phar-QA Quality in Pharmacy Education in Europe consortium and European Association of Faculties of Pharmacy (EAFP). Competency framework. Available from: http://eec-pet.eu/pharmacy-education/competencyframework/

9. Pharmaceutical Society of Australia. National Competency Standards Framework for Pharmacists in Australia. Deakin: PSA. 2010. Available from: https://www.psa.org.au/wp-content/uploads/2018/06/Competency_ standards_2010.pdf

10. Conselho Federal de Farmácia. Matriz de competências para a formação do farmacêutico na área de farmácia clínica. Brasilia: CFF. 2016. Available from: http://www.cff.org.br/userfiles/Matriz\%20final\%2018_11_2016\%20(site)(3).pdf

11. Conselho Federal de Farmácia. Competências para a atuação clínica do farmacêutico: Relatório do I Encontro Nacional de Educadores em Farmácia Clínica e Matriz de Competências para a Atuação: Brasília: CFF. 2017. Available from: www.cff.org.br/userfiles/file/Relatório\%20Enefar06jun2017_bx.pdf

12. National Association of Pharmacy Regulatory Authorities. Professional Competencies for Canadian Pharmacists at Entry to Practice. Ottawa: NAPRA. 2014. Available from: http://napra.ca/content_files/files/comp_for_ cdn_pharmacists_at_entrytopractice_march2014_b.pdf

13. The Pharmaceutical Society of Ireland. Core Competency Framework for Pharmacists. Dublin: PSI. 2013. Available from: https://www.thepsi.ie/ libraries/publications/psi_core_competency_framework_for_pharmacists. sflb.ashx

14. Pharmacy Council of New Zealand. Safe Effective Pharmacy Practice. Competence Standards for the Pharmacy Profession. Wellington: PCNZ. 2015. Available from: https://enhance2.psnz.org.nz/assets/downloads/ group_three/reflection/Standards_2015_FINAL.pdf

15. Ordem dos Farmacêuticos. Modelo de Competências Farmacêuticas da Ordem dos Farmacêuticos. 2016. Available from: http://www. ordemfarmaceuticos.pt /pt/formacao-continua/certificacao-de-competencias/

16. Singapore Pharmacy Council. Competency standards for pharmacists in Singapore (Entry to practice). Singapore: SPC. 2011. Available from: http:// www.healthprofessionals.gov.sg/content/dam/hprof/spc/docs/standard exam/SPC \%20Competency \%20Standards\%20Framework\%20(Feb\%20 2011),0.pdf

17. Orden CIN/2137/2008, de 3 de julio, por la que se establecen los requisitos para la verificación de los títulos universitarios oficiales que habiliten para el ejercicio de la profesión de Farmacéutico. BOE. 2008;(174):31692-5.

18. Royal Pharmaceutical Society. RPS Foundation Pharmacy Framework. A Framework for professional development in foundation practice across pharmacy. London: RPS. 2014. Available from: https://www.rpharms.com/ Portals/0/RPS\%20document\%20library/Open\%20access/Frameworks/ RPS\%20Foundation\%20Pharmacy\%20Framework.pdf 
19. American Association of Colleges of Pharmacy. Educational Outcomes 2013. Alexandria: CAPE. 2013.

20. Consejo General de Colegios Oficiales de Farmacéuticos. Directorio de Facultades de Farmacia Españolas. 2017. Available from: http:// www.portalfarma.com/profesionales/directorioenlaces/directorio/ facultadesfarmacia/Paginas/dirfacultadesfarma.aspx

21. Nunes-da-Cunha I, Martinez F, Fernandez-Llimos F. A Global Comparison of Internationalization Support Characteristics Available on College of Pharmacy Websites. Am J Pharm Educ. 2019;83(3):6592.

22. Koster A, Schalekamp T, Meijerman I. Implementation of Competency-Based Pharmacy Education (CBPE). Pharmacy. 2017;5(1):10.

23. Katoue MG, Schwinghammer TL. Competency-Based Pharmacy Education: An Educational Paradigm for the Pharmacy Profession to Meet Society's Healthcare Needs. In Pharmacy Education in the Twenty First Century and Beyond: Global Achievements and Challenges. Elsevier. 2018;169-88.

24. Nunes-da-Cunha I, Arguello B, Martinez FM, Fernandez-Llimos F. A Comparison of Patient-Centered Care in Pharmacy Curricula in the United States and Europe. Am J Pharm Educ. 2016;80(5):83.
25

Atkinson J, Rombaut B. The 2011 PHARMINE report on pharmacy and pharmacy education in the European Union. Pharm Pract. 2011;9(4):169-87.

26. Accreditation Council for Pharmacy Education. Accreditation standards and guidelines for the professional program in pharmacy leading to the doctor of pharmacy degree. Version 2.0. Chicago: ACPE. 2011. Available from: https:// www.acpe-accredit.org/pdf/S2007Guidelines2.0_ChangesIdentifiedInRed. pdf

27. Pittenger AL, Chapman SA, Frail CK, Moon JY, Undeberg MR, Orzoff JH. Entrustable Professional Activities for Pharmacy Practice. Am J Pharm Educ. 2016;80(4):57.

28. Haines ST, Pittenger AL, Stolte SK, Plaza CM, Gleason BL, Kantorovich A, et al. Core Entrustable Professional Activities for New Pharmacy Graduates. Am J Pharm Educ. 2017;81(1):S2.

29. Jarrett JB, Berenbrok LA, Goliak KL, Meyer SM, Shaughnessy AF. Entrustable Professional Activities as a Novel Framework for Pharmacy Education. Am J Pharm Educ. 2018;82(5):6256.

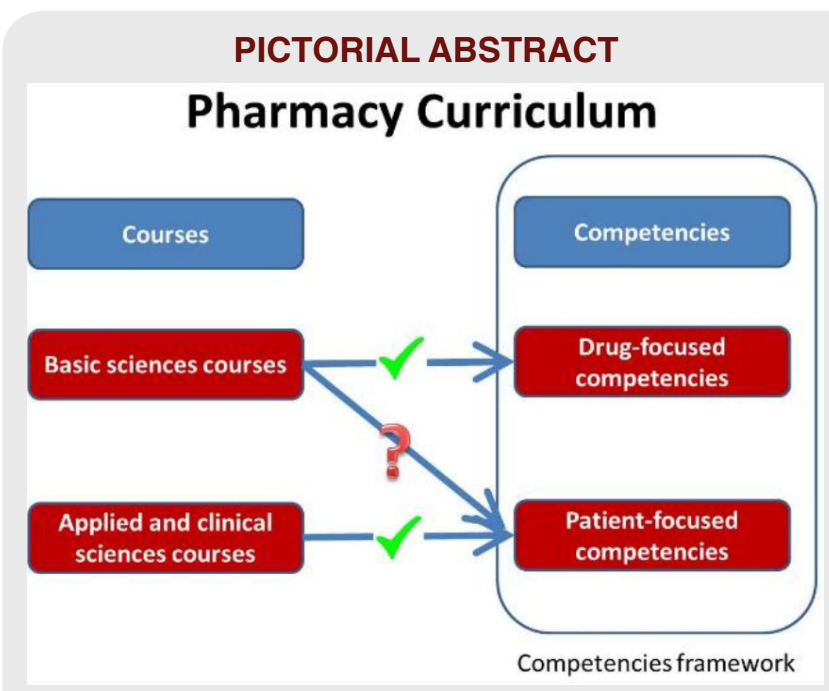

\section{SUMMARY}

This study was designed to investigate the incorrect use of the competencies frameworks, using all the Spanish higher institutions that provide the entry-level Pharmacy degree. The results confirm that Spanish official competencies framework, mandatory by law since 2008, is being incorrectly used. Basic and fundamental sciences disciplines are assigned to patientfocused competencies. It is postulated with this study that competencies should be paired to each topic of the course syllabus and not with the whole course.

\section{About Authors}

Ines Nunes-da-Cunha obtained her M.Sc. (Pharm) on Pharmaceutical care and her Ph.D. on Social Pharmacy at the University of Granada (Spain). She was Professor of Pharmaceutical Care and currently she is Community Pharmacist in Portugal.

Fernando Fernandez-Llimos obtained his Ph.D. on Drug Information at the University of Granada (Spain). He is Assistant Professor at the Department of Social Pharmacy, College of Pharmacy, University of Lisbon and Coordinator of the Pharmacoepidemiology and Social Pharmacy Group of the Institute for Medicines Research, University of Lisbon (iMed.ULisboa).

Cite this article: Nunes-da-Cunha I, Fernandez-Llimos F. Misuse of Competencies in Pharmacy Curriculum: The Spain Case Study. Indian J of Pharmaceutical Education and Research. 2019;53(4):620-8. 